\title{
Qualidade de vida de acadêmicos de enfermagem: análise à luz do modelo de Pender
}

\author{
Quality of life of nursing students: analysis in light of the Pender's model
}

\begin{abstract}
Como citar este artigo:
Lima RSS, Cavalcante JL, Machado MFAS, Cavalcante EGR, Quirino GS, Rebouças VCF. Quality of life of nursing students: analysis in light of the Pender's model. Rev Rene. 2020;21:e42159. DOI: https://doi.org/10.15253/2175-6783.20202142159
\end{abstract}

(D) Rosane Shirley Saraiva de Lima
(D)eane Lima Cavalcante
(D) Maria de Fátima Antero Sousa Machado ${ }^{1}$
(D) Edilma Gomes Rocha Cavalcante ${ }^{1}$
(D) Glauberto da Silva Quirino ${ }^{1}$
(D) Vitória de Cássia Félix Rebouças ${ }^{1}$

${ }^{1}$ Universidade Regional do Cariri. Crato, CE, Brasil.

\section{Autor correspondente: \\ Rosane Shirley Saraiva de Lima \\ Rua Vila Cruz, 393, Centro. \\ CEP: 63460-000. Pereiro, CE, Brasil. \\ E-mail: rosaneshirley15@gmail.com}

\begin{abstract}
RESUMO
Objetivo: compreender os estilos de vida dos acadêmicos de enfermagem à luz do Modelo de Promoção da Saúde Nola Pender. Métodos: estudo qualitativo, realizado com 11 acadêmicos de enfermagem por meio de um grupo focal a partir de um roteiro semiestruturado. Obtiveram-se duas categorias analíticas: Características e experiências individuais; Sentimentos e conhecimentos sobre o comportamento que se quer alcançar. Resultados: como principais fatores que repercutem na qualidade de vida os acadêmicos relataram baixa qualidade e pouco tempo de sono, sedentarismo e alimentação inadequada, que estão relacionados à rotina universitária. Conclusão: a utilização do referido Modelo foi importante para identificar os fatores que repercutem nos estilos de vida dos acadêmicos, em seus aspectos biopsicossocial e suas correlações, demonstrando a sua relevância ao apontar a necessidade de intervenções que atendam a sua realidade.

Descritores: Promoção da Saúde; Enfermagem; Teoria de Enfermagem; Qualidade de Vida.
\end{abstract}

\begin{abstract}
Objective: to understand the lifestyles of nursing students in the light of the Nola Pender's Health Promotion Model. Methods: qualitative study conducted with 11 nursing students through a focus group using a semi-structured script. Two analytical categories were obtained: Individual characteristics and experiences; Feelings and knowledge about the behavior to be achieved. Results: the short time and poor quality of sleep, sedentary lifestyle and inadequate diet, which are related to the university routine, were reported by the academics as main factors that affect quality of life. Conclusion: the use of this model was important to identify the factors that affect the students' lifestyles, biopsychosocial aspects and their correlations, demonstrating its relevance in pointing out the need for interventions that meet their reality.

Descriptors: Health Promotion; Nursing; Nursing Theory; Quality of Life.
\end{abstract}




\section{Introdução}

A concepção sobre qualidade de vida é complexa, sem definição consensual e envolve percepções individuais e subjetivas relacionadas ao que cada um compreende como saúde. Tendo em vista o seu conceito ampliado, defende-se que ter qualidade de vida envolve o equilíbrio dos aspectos biopsicossociais ${ }^{(1-2)}$.

Nesse sentido, ter qualidade de vida permeia a autonomia nas escolhas dos indivíduos atrelada às condições em que vivem e trabalham. Assim, inúmeras são as situações que podem influenciar na qualidade de vida do ser humano, sendo uma delas o ingresso à universidade, visto que esta é um espaço onde as pessoas permanecem durante um período importante de suas vidas, fundamental no desenvolvimento humano e profissional do indivíduo, contudo demanda mudanças em sua rotina que afetam o seu estilo de vida, o que pode repercutir no seu desempenho acadêmico ${ }^{(3)}$.

Ao adentrar na universidade, o estudante, em sua maioria jovem, encontra-se em fase de transição para a vida adulta, trazendo consigo várias inseguranças, como a sua adaptação à academia, a ansiedade de satisfazer as expectativas sociais e familiares, e no decorrer dos estudos o estresse com as muitas atividades e compromissos acadêmicos, o que será enfrentado de forma relativa à maturidade psicológica de cada um. Assim sendo, toda essa nova rotina pode trazer desgastes físicos e mentais, comprometendo, desse modo, a sua aprendizagem ${ }^{(4)}$.

Nesse sentido, a enfermagem, corpo profissional que estabelece maiores relações com as pessoas, em que seu cuidado está intrinsecamente relacionado ao seu bem-estar e promoção da saúde, deve capacitar os estudantes para a adoção de escolhas saudáveis, tendo em vista o impacto dessas escolhas no surgimento de problemas, por exemplo, o seu rendimento acadêmico, diminuição do absenteísmo, dentre outros que circundam esse contexto ${ }^{(5)}$.

Para alicerçar a prática de enfermagem e auxiliar na compreensão da complexidade do processo saúde-doença, é possível utilizar modelos e teorias de enfermagem. Estas propiciam o embasamento científico ao cuidado de enfermagem, possibilitando um entendimento de maior amplitude sobre os fenômenos que os enfermeiros se defrontam, contribuindo também para a construção do conhecimento na área da enfermagem ${ }^{(6)}$

Entre aquelas voltadas à promoção da saúde, inclui-se o Modelo de Nola Pender, o qual foi desenvolvido nos EUA, aproximadamente nos anos de $1980^{(7)}$. É amplamente utilizado como arcabouço de pesquisas que objetivam a previsão de estilos de vida e comportamentos específicos promotores de saúde. É uma teoria de médio alcance e seus grandes conceitos são: ambiente, saúde, enfermagem e pessoa ${ }^{(8)}$.

Seus grandes componentes e variáveis são: 1) Características e experiências individuais: abrange o comportamento anterior, ou seja, os hábitos passados do indivíduo e que devem ser modificados e os fatores pessoais divididos entre biológicos, psicológicos e socioculturais; 2) Sentimentos e conhecimentos sobre o comportamento que se quer alcançar: percepção de benefícios para ação, ou seja, as representações mentais positivas que reforçam a adoção de determinado comportamento; percepção de barreiras para ação: percepções negativas no que concerne à disponibilidade, conveniência, despesa, natureza ou consumo de tempo, danos, custos pessoais e dificuldades para determinada ação ${ }^{(7)}$.

Esse segundo componente compreende também percepção de autoeficácia: julgamento do indivíduo sobre a sua capacidade pessoal e habilidades para organizar e realizar uma determinada ação; sentimentos em relação ao comportamento: exprime as emoções positivas ou negativas, agradáveis ou desagradáveis $^{(7)}$; repercussões interpessoais: comportamentos, interesses, crenças ou atitudes de outras pessoas que podem interferir no seu comportamento como família, colegas, cônjuge prestadores de cuidados de saúde e normas sociais; e repercussões situacionais: situações do ambiente em que as pessoas vivem podem afetar diretamente os comportamentos ${ }^{(7)}$; 3) Resultados do comportamento: compreende o compromisso 
com o plano de ação, que se pauta nas ações propostas que propiciaram ao indivíduo o alcance e mantenham condutas promotoras de saúde esperadas a partir das intervenções de enfermagem.

O compromisso com o plano estabelecido envolve comunicação para realizar uma ação específica em um determinado momento e local, e com pessoas específicas ou sozinhas, independentemente de preferências concorrentes (que são aquelas contrárias ao comportamento de saúde e identificação de estratégias decisivas para reforçar o comportamento que podem contemplar as exigências e preferências com necessidade de modificação imediata). Isso resultará no comportamento de promoção da saúde ${ }^{(7)}$. No presente estudo, utilizaram-se os dois primeiros componentes, considerando que não foram implementadas intervenções.

Pretende-se com esta pesquisa defender a necessidade do acompanhamento e avaliação do estilo e qualidade de vida dos estudantes universitários para que venham a ser planejadas intervenções visando promover a saúde desse público e o apoio ao enfrentamento das suas dificuldades no percurso estudantil ${ }^{(9)}$. Neste estudo, tratou-se em especial dos acadêmicos de enfermagem. Nessa direção, o objetivo do estudo foi compreender os estilos de vida dos acadêmicos de enfermagem à luz do Modelo de Promoção da Saúde Nola Pender.

\section{Métodos}

Estudo qualitativo, realizado em uma universidade pública estadual, do Nordeste brasileiro, no interior do estado do Ceará. Dos 21 discentes do curso de graduação de enfermagem, participaram do estudo 11 que atenderam ao seguinte critério de inclusão: estar devidamente matriculado no oitavo semestre do curso de Enfermagem. Excluíram-se os discentes que não compareceram para a realização do grupo focal. A escolha pelo semestre deveu-se ao fato de os acadêmicos terem cumprido mais da metade do periódo de integralização do curso (10 semestres), ou seja, passado um maior tempo no ambiente universitário.

Os discentes foram contatados em sala de aula e convidados formalmente pelas pesquisadoras para participação no estudo; após o aceite, agendou-se o encontro para realização do grupo focal. 0 grupo contou com a presença de um moderador e um observador, com sessão de, aproximadamente, 60 minutos que ocorreu em uma sala de aula reservada do próprio curso de enfermagem com ventilação, luminosidade e acuidade adequadas. Utilizou-se configuração circular, que possibilitou a integração dos participantes, com espaço para organização de coffee break e discussões coletivas. 0 estudo norteou-se pela seguinte indagação: Quais fatores repercutem na qualidade de vida de acadêmicos de enfermagem?

A técnica de grupo focal permite a integração de um grupo pequeno e homogêneo, para discutir sobre um determinado assunto, e avalia um tema proposto, na perspectiva de apreender percepções, opiniões, atitudes e representações sociais ${ }^{(10)}$. Buscaram-se nas falas dos estudantes e com base no referencial teórico adotado no estudo as características e experiências individuais e os sentimentos e conhecimentos sobre o comportamento que se quer alcançar.

O grupo focal foi realizado em um único momento, outubro de 2018, e antes da entrevista em grupo, solicitou-se que os participantes respondessem por escrito sobre os dados sociodemográficos. 0 encontro foi gravado em áudio, conforme autorização dos participantes, e submetido à transcrição na íntegra. Estes foram codificados para garantir o anonimato, com as seguintes denominações: Estudante 1, Estudante 2 e assim sucessivamente. A participação foi voluntária, mediante assinatura do Termo de Consentimento Livre e Esclarecido.

Após a transcrição das falas resultantes do grupo focal, os dados obtidos foram organizados e analisados a partir da análise de conteúdo ${ }^{(11)}$, a qual é dividida em três fases: 1) pré-análise: envolve a compreensão das informações coletadas a partir da leitura dos dados e elaboração das hipóteses e objetivos, interpretação das informações com elaboração 
de indicadores; 2) Exploração do material: consiste no recorte do material coletado em unidades de registro (palavras, frases ou parágrafos) por meio das quais se identificam as palavras-chaves atarvés do agrupamento de temas e falas relacionadas à categorização; 3) Interpretação: compreende o tratamento dos resultados capitando os conteúdos das falas de acordo com o referencial teórico.

Para a realização da pesquisa, respeitaram-se os aspectos éticos da Resolução 466/2012 do Conselho Nacional de Saúde, que dispõe sobre o envolvimento direto ou indireto com seres humanos. 0 presente estudo faz parte de um projeto maior, do tipo guarda-chuva ou de referência, intitulado Estudos sobre promoção da saúde nos ambientes educacionais e de trabalho, aprovado pelo Comitê de Ética em Pesquisa da Universidade Regional do Cariri através do no 1.973.784/2017 e Certificado de Apresentação e Apreciação Ética no 65520617.0000.5055.

\section{Resultados}

Participaram da pesquisa dez estudantes do sexo feminino e um do sexo masculino. A idade dos jovens variou entre 20 e 26 anos (fatores biológicos), seis se consideravam brancos e cinco pardos. Com relação à renda, quatro declararam uma média de dois a quatro salários mínimos e sete declararam possuir renda menor que um salário mínimo $(\mathrm{R} \$ 954,00)$, fato este melhor delineado posteriormente por meio das suas falas (fatores socioculturais).

Em relação à moradia, cinco moravam com os pais, dois com os amigos e quatro com outros familiares. Para identificar previamente algumas condutas perguntou-se sobre a prática de atividade física, em que apenas um afirmou praticar.

Apresentam-se, a seguir, os resultados advindos do corpus descritivo resultante das falas dos discentes no grupo focal organizadas em duas categorias: Experiências individuais; e Sentimentos e conhecimentos sobre o comportamento que se quer alcançar.

\section{Categoria 1. Experiências individuais}

Esta categoria apresenta os comportamentos anteriores, ou seja, os hábitos de vida dos participantes que não favorecem a sua qualidade de vida e precisam ser modificados, pois podem interferir em possíveis comportamentos promotores de saúde. Além disso, expressa os fatores pessoais, definidos como biológicos, psicológicos e socioculturais, que predispõem a determinadas condutas.

Para correlacionar esses dados e compreender os fatores que interferem na qualidade vida, segundo a percepção dos estudantes, buscou-se indagar primeiramente o que os acadêmicos entendiam sobre o que é ter qualidade vida, sendo esta a pergunta disparadora do grupo focal. Os participantes declararam que qualidade de vida é algo subjetivo, ou seja, está relacionada às experiências individuais de cada pessoa, mas que, em linhas gerais, relaciona-se ao conceito ampliado de saúde da Organização Mundial da Saúde e ações de promoção da saúde: Qualidade de vida diz respeito ao bem-estar psicossocial, econômico (Estudante 1). Vai além da ausência de doenças (Estudante 2). Cada um tem aquilo que acredita ser qualidade de vida. Por exemplo, pra mim qualidade de vida está em fazer atividade física, ou então estar em um grupo de amigos, já pode ser ir pra um cinema, fazer outras coisas diversas (Estudante 4). A qualidade de vida, ela também vai tá tanto das questões individuais, é de como ele está inserido naquele grupo social e quais são as condições também que aquele local oferece pra ele (Estudante 5). Aí entra a promoção da saúde, que são ações voltadas ao âmbito social, político, que visam desenvolver essa qualidade de vida. Ele dá ferramentas pra que a pessoa, a população, comunidade, tenha subsídios de se promover sua própria qualidade de vida (Estudante 3).

Para identificar suas condutas prévias com necessidade de modificação, os participantes foram questionados sobre quais os seus hábitos de vida que interferem na sua qualidade de vida. Seus relatos apontaram baixa qualidade e pouco tempo de sono, sedentarismo e alimentação desequilibrada: Alimentação errada, às vezes o almoço é um salgadinho e não toma café da manhã (Estudante 2). As horas de sono, de repouso, de estudo, que 
a gente poderia ter extra, a gente tem que se virar na manhã e na madrugada, então isso interfere muito na nossa qualidade de vida (Estudante 4). Falta de exercício físico (Estudante 7).

Com relação aos fatores psicológicos, constataram-se estresse, baixa autoestima e ansiedade, que denotam estar relacionados à rotina acadêmica: Às vezes tinha alguma coisa da minha família e eu não ia, eu estava fazendo o trabalho, mas eu estava estressada por dentro por eu não tá lá naquele momento, nem isso estava fazendo bem para meus estudos, acabava não me concentrando direito. Fora a autoestima porque quando tá perto de prova, eu não consigo acordar e me arrumar (Estudante 7). No começo eu só me detia à faculdade como experiência própria eu adquiri uma ansiedade quando chegava o final do semestre era hospital, hospital na certa. Por causa dessa ansiedade: Será que vai dar certo? Será que eu vou passar para o próximo (semestre). A gente tem essa pressão tanto da sociedade como familiar (Estudante 9).

\section{Categoria 2. Sentimentos e conhecimentos sobre o comportamento que se quer alcançar}

Esta categoria permitiu a compreensão dos fatores que levavam os acadêmicos à adoção dos hábitos de vida identificados, bem como dos fatores emocionais e cognitivos envolvidos em um possível processo de mudança para comportamentos promotores de saúde, a partir das seguintes variáveis: percepção de benefícios para ação, percepção de barreiras para ação, percepção de autoeficácia, sentimentos em relação ao comportamento, interpessoais e repercussões situacionais.

Os acadêmicos reconheceram como benefícios que podem ser alcançados a partir de mudanças em seus hábitos: emocionais, o controle de índices de gordura, melhora no condicionamento e diminuição à predisposição às doenças: No emocional, melhorar a qualidade de vida, diminuir, por exemplo, índice de triglicerídeos, colesterol, a partir do momento que você consegue conciliar a faculdade e sair. É totalmente diferente, porque você dedica um pouco do seu tempo pra cada coisa e isso aí faz com que o estresse diminua (Estudante 2). Melhorar até mesmo condicionamento (Estudante 3). Musculação, né?
Eu acho que vai evitar muita coisa das predisposições genéticas que tem na minha família, em relação a hipertensão, diabetes e câncer de mama (Estudante 8).

Quanto às barreiras para realizar essas ações, relataram falta de foco, tempo, determinação e questão financeira: Falta de foco, falta de tempo, falta de determinação (Estudante 2). Gasto financeiro (Estudante 10). Eu acho que a gente vai tudo procrastinando, porque, assim, a gente joga a culpa na graduação, aí depois vem um Mestrado ou então um casamento, não sei... vai vir uma residência, um filho, não sei...e aí você sempre vai jogar culpa, vai culpabilizar alguma coisa (Estudante 11).

Com relação à percepção de autoeficácia, mencionaram que não conseguem se organizar para realizar mudanças em seus hábitos no momento pelo tempo restrito e por não saber gerenciá-lo, cansaço e falta de esforço: É muita coisa ao mesmo tempo acontecendo e com pouco tempo, assim que acaba da gente não conseguir gerenciar tudo. A vontade é dez, mas quando a gente tem um dez, a gente vai pra prática. A gente vai um dia, entendeu? (Estudante 5). Eu não mudo, também eu vejo que é falta de esforço minha, porque nem que fosse a noite dá pra fazer uma caminhada, uma academia ou sei lá. Só que as vezes o cansaço, a rotina, você acaba não indo (Estudante 2).

Quanto a como se sentiam em relação ao comportamento, os participantes pontuaram ter a sensação de impotência e se sentirem mal por não conseguirem conciliar hábitos saudáveis à rotina estudantil, ainda mais por não conseguirem cumprir as orientações que fazem aos usuários em ambiente de estágio: Pelo menos eu me sinto impotente pelo fato da faculdade, eu me sinto impotente por não conseguir ter uma alimentação saudável, não conseguir realizar pelo tempo. Eu fazia atividade física e parei (Estudante 1). Eu me sinto mal, porque, tipo, eu estudo todo o dia aqui na faculdade a importância e tudo, mas quando chego em casa não adianta, eu gosto de comer fritura, comer coisa salgada, e mesmo eu sabendo, é comendo e pensando, que faz mal pra mim, mas eu estou... eu não mudo (Estudante 2). E pra nós do curso de enfermagem é mais complicado ainda porque a gente faz essas orientações, a gente prega tudo isso e acaba que a gente não consegue cumprir (Estudante 3).

No que concerne às repercussões interpessoais, os jovens destacaram a influência dos hábitos dos familiares e amigos, bem como a necessidade de adaptá-los em decorrência de alguma doença de 
membros da família ou amigos, principalmente na questão alimentar: Minha mãe, ela gosta muito de fruta, ela só não faz atividade física, gosta de uma alimentação saudável, se for depender dela, né, mas aqui na faculdade não tem como (Estudante 7). Minha avó tem diabetes, e aí faz uns três meses que acho que ela fez uns exames de glicose, e aí deu alterado e ela precisou realmente modificar os hábitos em relação ao açúcar e lá em casa, questão é que todo mundo agora é adoçante e açúcar (Estudante 1). Meu amigo também tem doença renal crônica e aí, ele não pode comer nada. Nós estamos mudando os nossos hábitos pra poder incluir ele no nosso convívio social e em nossa família (Estudante 9).

No que diz respeito às repercussões situacionais, pôde-se identificar rotina universitária, faculdade em tempo integral, atividades extracurriculares, morar longe dos pais, morar sozinho, situação socioeconômica em decorrência de não trabalhar e depender dos pais: A questão de alimentação também pra muita gente que mora longe dos pais, porque você chega em casa e tem que se virar o mais rápido que você puder pra voltar (Estudante 8). O tempo pra você fazer também, pra preparar essa comida, essa alimentação, também é muito restrito, principalmente porque a faculdade é tempo integral e acaba que a gente tem menos tempo. Então quem tem que chegar em casa, é pra estudar. Quem mora só, acaba tendo que fazer comida (Estudante 1). Então, além das coisas da faculdade, a gente ainda tem as coisas de grupo de pesquisa (Estudante 7). Eu acho que a questão financeira influi muito na nossa questão é porque temos que ficar na universidade o dia todo, tempo integral, então a gente não tem como ter um emprego, quem não mora com os pais, precisam vir de outras cidades e dependem dos pais para pagar um aluguel aqui, pra alimentação (Estudante 9).

\section{Discussão}

Considerou-se como limitação deste estudo a implementação de dois componentes propostos pelo Modelo de Promoção da Saúde ${ }^{(7)}$, o que aponta a necessidade de intervenção e avaliação dos resultados comportamentais. Contudo, o conhecimento adquirido com esta investigação poderá subsidiar o planejamento de intervenções que promovam a saúde de estudantes universitários, tendo em vista as suas demandas biopsicossociais.
Nesta pesquisa, os discentes relataram sobre os fatores que podem repercutir no seu estilo de vida à luz do Modelo de Promoção da Saúde ${ }^{(7)}$. Pôde-se identificar em linhas gerais que os principais fatores estão relacionados à rotina universitária, por exemplo, uma alimentação desequilibrada, sedentarismo, estresse, ansiedade e baixa autoestima.

No que tange às características e experiências individuais, um estudo realizado com 146 estudantes de enfermagem de uma universidade pública do Sul do Brasil constatou que 89,0\% dos acadêmicos de enfermagem eram da população feminina e 54,1\% relataram ser sedentários ${ }^{(12)}$.

Em relação aos hábitos não saudáveis, como alimentação desequilibrada, sono prejudicado e relações sociais e familiares alteradas, estes são ocasionados devido aos compromissos acadêmicos, o que produz ansiedade, estresse e baixa autoestima. Resultado similar, em uma Universidade Estadual de Mato Grosso do Sul, também apontou que o envolvimento nos compromissos acadêmicos ocasiona ansiedade e estresse entre os estudantes ${ }^{(4)}$. 0 estresse é um fator nocivo à saúde mental e ao bem-estar físico, estando associado a doenças como as cardiovasculares, câncer e distúrbios gastrointestinais, assim como depressão, padrões de sono ruins e incapacidade de realizar atividades diárias eficazes ${ }^{(7)}$.

Assim, hábitos de vida saudáveis, como uma nutrição adequada, são determinantes para uma boa saúde. Uma avaliação detalhada determina quais intervenções são mais satisfatórias para melhorar o estado nutricional de graduandos de enfermagem ${ }^{(13)}$.

De acordo com o modelo teórico utilizado, cada pessoa tem características e experiências pessoais únicas que vão afetar as suas ações subsequentes. A importância do seu efeito será relativa ao comportamento alvo que se deseja modificar, pois a sua frequência e como esse hábito está arraigado à vida do indivíduo, bem como o mesmo vê as possibilidades e dificuldades para a mudança, implicarão na probabilidade de que o mesmo se engaje em comportamentos promotores de saúde ${ }^{(7)}$. 
A relação entre saúde e prática de atividade física é considerada relevante para a promoção da saúde e melhoria da qualidade de vida, uma vez que previne doenças, bem como ajuda a manter hábitos saudáveis em todas as etapas do ciclo de vida ${ }^{(14)}$.

Dessa forma, cabe no espaço das instituições do ensino superior a incorporação de práticas de promoção da saúde, como atividades físicas voltadas aos estudantes no ambiente universitário, assim como no estudo sobre Estilo de vida e fatores associados entre estudantes universitários, o qual confirmou que são evidentes os riscos relacionados ao estilo de vida aos quais os estudantes estão expostos, assim a importância do desenvolvimento de medidas educativas que estimulem a prática de hábitos saudáveis de vida(a ${ }^{(15)}$.

No que se refere aos sentimentos e conhecimentos sobre o comportamento que se quer alcançar, os estudantes perceberam os seguintes benefícios a partir de mudanças em seus hábitos: emocionais, o controle de índices de gordura, melhora no condicionamento e diminuição à predisposição às doenças. É importante ressaltar que estilos de vida saudáveis devem ser adotados e mantidos principalmente por acadêmicos da saúde, em especial da enfermagem, já que sua profissão exige um bom estado de saúde físico e mental para cuidar do outro, além de estimular outros indivíduos a conseguirem uma vida mais adequada ${ }^{(16)}$.

Quanto às barreiras para realizar as ações de mudanças de hábitos e percepção de autoeficácia, relataram a falta de foco, tempo, determinação e questão financeira. Nesse contexto, faz-se necessário que se busque, em um possível plano de ação, elaborar estratégias que propiciem aos estudantes o enfrentamento dessas dificuldades para que alcancem a qualidade de vida $^{(17)}$.

O Modelo de Promoção da Saúde propõe que a autoeficácia percebida é influenciada pelo afeto relacionado à atividade, ou seja, quanto mais positivo o afeto, maiores são as percepções de eficácia, ou seja, essa relação deve ser recíproca. Assim sendo, a percepção de autoeficácia influencia no enfrentamento às barreiras percebidas para a implementação da ação.
Desse modo, sentir-se eficaz e qualificado motiva o comportamento de promoção da saúde e um possível compromisso ou persistência na busca de um plano de ação ${ }^{(7)}$.

Observa-se no presente estudo a necessidade de que a instituição universitária elabore estratégias que busquem ajudar o estudante quanto à mudança dos seus hábitos de vida, para isso é necessário que, além de ambientes para prática de atividades físicas, seja possível fornecer alimentos saudáveis e com valores acessíveis para o consumo dos alunos.

No que concerne ao comportamento, os acadêmicos relataram sentimentos negativos e de impotência por não conseguirem conciliar hábitos saudáveis com a rotina estudantil, ainda mais por não conseguirem cumprir as orientações que fazem aos usuários em ambiente de estágio. Resultados semelhantes foram encontrados em outro estudo, no qual os discentes relataram o mesmo sentimento dos estudantes em relação a essa contradição por não colocarem em prática o que orientam aos seus usuários em campo de estágio $^{(12) .}$

Segundo o Modelo, todo comportamento também é acompanhado por emoções ou problemas, afeto positivo ou negativo. 0 comportamento prévio é proposto para moldar todas essas cognições e afetos específicos. Intervenções nesse âmbito devem auxiliar os indivíduos a moldarem uma história comportamental positiva para o futuro, concentrando-se nos benefícios de um comportamento, ensinando como ultrapassar obstáculos para realizar o comportamento e atingir altos níveis de eficácia e afeto positivo por meio de experiência de desempenho bem-sucedida e feedback positivo ${ }^{(7)}$.

Para os estudantes do estudo em tela, com relação às repercussões interpessoais, seus familiares e amigos tinham função importante sobre os seus hábitos de vida. De acordo com o Modelo de Promoção da Saúde, essa situação interfere em comportamentos promotores de saúde e no estímulo à adesão de um possível plano com ações a serem implementadas, pois as pessoas são sensíveis aos desejos, exemplos e 
elogios dos outros, embora haja variações na extensão dessa influência é relevante para reforçar comportamentos saudáveis, como podem também dificultar a adoção do comportamento de acordo com o contexto socioeconômico e cultural. Além disso, os indivíduos são levados a desempenharem com mais competência quando inseridos em ambientes que considerem compatíveis a si e nos quais se sentem seguros. Ainda, reforça-se que a família possui uma influência recíproca entre seus membros, confirmando-se como uma importante estrutura social primária para a promoção da saúde ${ }^{(7)}$.

Em referência às repercussões situacionais, pôde-se identificar rotina universitária, faculdade em tempo integral, atividades extracurriculares, morar longe dos pais, morar sozinho, situação socioeconômica em decorrência de não trabalhar e depender dos pais. Assim, é importante que as instituições de ensino superior revejam as questões da carga horária, bem como as atividades extracurriculares, adequando-se à condição de vida dos estudantes ${ }^{(18)}$.

As repercussões situacionais segundo o Modelo interferem direta e indiretamente no comportamento de saúde, além disso, são consideradas uma chave importante para o desenvolvimento de estratégias novas e mais eficazes para facilitar a aquisição de comportamentos de promoção da saúde em diferentes populações. Essa avaliação realizada a partir do Modelo pode fornecer informações valiosas para o desenvolvimento de estratégias de aconselhamento, ajudar as pessoas a mudarem o comportamento negativo ou a adotarem um novo comportamento saudável ${ }^{(7)}$.

Diante do exposto, vê-se a necessidade de intervenções para aumentar as expectativas de eficácia e para compreender como o conceito interage com outros determinantes motivacionais para melhorar os comportamentos de saúde. Além disso, o modelo pauta-se nas ações propostas que propiciaram que o indivíduo alcance e mantenha o comportamento de promoção da saúde esperado.

\section{Conclusão}

Observou-se que o ambiente acadêmico, correlacionado às condições socioeconômicas, individuais, pessoais e sociais, influenciam significativamente no seu estilo de vida, interferindo na sua qualidade de vida. Ter acesso a esses dados identificados por meio do referencial adotado auxilia no planejamento das medidas necessárias para a mudança com vista à promoção da sua saúde a partir da compreensão das relações causais das condições do seu cotidiano com a adoção de hábitos de vida saudáveis ou não. Infere-se que o Modelo de Pender torna-se necessário para a prática de enfermagem, sendo recomendado pelos resultados apresentados.

\section{Colaborações}

Lima RSSL e Cavalcante JL contribuíram com a coleta, análise, interpretação dos dados e redação do artigo. Machado MFAS contribuiu com a concepção do projeto, análise dos dados e redação do artigo. Cavalcante EGR contribuiu com a análise, interpretação dos dados e redação do artigo. Quirino GS contribuiu com a revisão crítica relevante do conteúdo intelectual e redação do artigo. Rebouças VCF contribuiu na análise e interpretação dos dados e aprovação final da versão a ser publicada.

\section{Referências}

1. Machado DGS, Silva VS, Nascimento Neto LI, Farias Júnior LF, Fonteles AI, Agrícola PMD, et al. Exercício físico em academia, qualidade de vida e satisfação coma saúde. Rev Bras Qual Vida. 2015; 7(4):269-78. doi: http://dx.doi.org/10.3895/ rbqvv7n 4.3265

2. Moura IH, Nobre RS, Cortez RMA, Campelo V, Macêdo SF, Silva ARV. Quality of life of undergraduate nursing students. Rev Gaúcha Enferm. 2016; 37(2):e55291. doi: http://dx.doi. org/10.1590/1983-1447.2016.02.55291 
3. Diez OJ, López RNO. Estudiantes Universitarios y el estilo de vida. Rev Ibero-am Producón Acad Gestión Educ [Internet]. 2017 [cited Jul 13, 2019];4(8):1-15. Available from: http://www.pag. org.mx/index.php/PAG/article/view/723/1009

4. Araujo MAN, Lunardi Filho WD, Leite LRC, Ma RTK, Silva AA, Souza JC. Quality oflife of nursing students. Rev Rene. 2014; 15(6):990-7. doi: http://dx.doi. org/10.15253/2175-6783.2014000600012

5. Soares AM, Pereira MAS, Canavarro JMAP. Promoção da Saúde nas Instituições de Ensino Superior Portuguesas: reflexões e desafios. Rev Port Pedag. 2015; 49(2):115-37. doi: http:// dx.doi.org/10.14195/16478614_492_6

6. Silva NRF, Farias DC, Sousa JR, Bezerra FMC. Ferreira LS, Carvalho PMG. Teorias de enfermagem aplicadas no cuidado a pacientes oncológicos: contribuição para prática clínica do enfermeiro. Rev UNINGÁ [Internet]. 2018 [citado 2019 Jun 13]; 55(2):59-71. Disponível em: http://revista. uninga.br/index.php/uninga/article/view/1385

7. Pender NJ, Murdaugh C, Parsons MA. Health promotion in nursing practice. Englewood Cliffs: Prentice Hall; 2015.

8. Mcewen M, Wills EM. Bases teóricas de enfermagem. Porto Alegre: Artmed; 2016.

9. Gama ASM. Qualidade de vida de estudantes de enfermagem do Amazonas, Brasil. Rev Baiana Enferm. 2016; 30(4):1-9. doi: http://dx.doi. org/10.18471/rbe.v30i4.17011

10. Minayo MCS. O desafio do conhecimento: pesquisa qualitativa em saúde. São Paulo; Hucitec; 2013.

11. Bardin L. Análise de conteúdo. São Paulo; Edições 70; 2016.

12. Hirsch CD, Barlem ELD, Almeida LK, Barlem JGT, Lunardi VL, Ramos AM. Factors perceived by nursing students as triggers of stress in the training environment. Texto Contexto Enferm. 2018; 27(1):e0370014. doi: http://dx.doi. org/10.1590/0104-07072018000370014
13. Maniglia FP, Santos DM, Oliveira FCM, Ribeiro JC. Avaliação do consumo alimentar e estado nutricional de graduandos em enfermagem. Rev Int Estudos Saúde [Internet]. 2018 [citado 2019 jun 18]; 7(2):51-9. Disponível em: https:// periodicos.uniarp.edu.br/index.php/ries/article/ view/1397

14. Ferreira JS, Diettrich SHC, Pedro DA. Influência da prática de atividade física sobre a qualidade de vida de usuários do SUS. Rev Saúde Debate. 2015; 39(106):792-801. doi: http://dx.doi. org/10.1590/0103-1104201510600030019

15. Guimarães MR, Batista AMO, Santos IMS, Vale MPSS, Moura IH, Silva ARV. Lifestyle and associated factors between university students. Rev Enferm UFPE on line [Internet]. 2017 [cited Jun 13, 2019; 11(8):3228-35. doi: Available from: https:// periodicos.ufpe.br/revistas/revistaenfermagem/ article/viewFile/110188/22073

16. Freitas AMC, Bárbara JFRS, Vale PRLF, Carvalho SS, Carvalho LF, Nery GS. Perceptions of nursing studentsregardingthequality oflifein the academic career. Rev Enferm UFSM. 2017; 7(2):152-66. doi: http://dx.doi.org/10.5902/2179769225391

17. Assis PYS, Pereira DL, Lima CA, Vieira MA, Costa FM. Qualidade de vida de estudantes da graduação em enfermagem: revisão de literatura. Rev Eletr Gestão Saúde [Internet]. 2014 [citado 2019 jun 13]; 5(3):2115-36. Dipsonível em: https://dialnet. unirioja.es/servlet/articulo?codigo $=5556073$

18. Freitas ACM, Malheiros RMM, Lourenço BS, Pinto FF, Souza CC, Almeida ACL. Intervening factors in the quality of life of nursing students. Rev Enferm UFPE on line [Internet]. 2018 [cited Jun 13, 2019]; 12(9):2376-85. Available from: https:// periodicos.ufpe.br/revistas/revistaenfermagem/ article/view/230110/29923

\section{(cc) BY}

Este é um artigo de acesso aberto distribuído sob os termos da Licença Creative Commons 